

\title{
Análisis situacional de salud de diferentes sectores del
}

País

\section{Situational health analysis of different sectors of the country}

\author{
Análisis situacional de salud
}

\begin{abstract}
Virginia Esmeralda Pincay Pin
Universidad Estatal del Sur de Manabí; virginia.pincay@unesum.edu.ec; https://orcid.org/0000-0001-87765433.
\end{abstract}

\section{Contacto: virginia.pincay@unesum.edu.ec}

Recibido: 29-09-2019

Aprobado: 13-12-2019

\section{Resumen}

El Análisis Situacional de Salud es un proceso de diagnóstico establecido por las autoridades del sistema de salud para recopilar información pertinente en una población determinada para así conocer información referente a la situación de las enfermedades que determinan el estado de salud, a pesar de los esfuerzos del Ministerio de Salud Pública por brindar una atención más integral y con acceso para todos, aún quedan muchos lugares donde es necesario aplicar herramientas de diagnóstico situacional para conocer la realidad en la que viven los ciudadanos y conocer sus determinantes que afectan la calidad de vida. El objetivo fue elaborar un diagnóstico situacional de un conjunto de familias de los estudiantes tercer semestre carrera enfermería de los diferentes sectores del Ecuador, identificando los principales factores de riesgos que presentan las familias a su vez describir el perfil epidemiológico y diseñar un plan enfocado en la prevención y mitigación de los factores de riesgo y enfermedades detectadas en la población de estudio. La metodología utilizada en la investigación es de tipo descriptiva, la información bibliográfica se obtuvo fuentes confiables como tesis, libros, artículos científicos y en diferentes bases de datos y la utilización de la ficha familiar. Conclusión; los principales factores de riesgo identificados en este grupo de familias fueron algunas enfermedades; alergias, diabetes, hipertensión, debido a emisiones contaminantes y condiciones del entorno, inadecuada alimentación, riesgos sanitarios, presencia de industrias, estos riesgos se podrían disminuir con los cuidados adecuados.
Palabras claves: Atención primaria, enfermería comunitaria, ficha familiar, factores de riesgos en salud.

\begin{abstract}
The Health Situation Analysis is a diagnostic process established by the authorities of the health system to collect relevant information in a given population in order to know information regarding the situation of diseases that determine the state of health, despite the efforts of the Ministry of Public Health for providing more comprehensive care and with access for all, there are still many places where it is necessary to apply situational diagnosis tools to know the reality in which citizens live and know its determinants that affect the quality of life. The objective was to develop a situational diagnosis of a set of families of third semester nursing students from different sectors of Ecuador, identifying the main risk factors presented by families, in turn, describe the epidemiological profile and design a plan focused on the prevention and mitigation of risk factors and diseases detected in the study population. The methodology used in the research is descriptive, the bibliographic information was obtained from reliable sources such as thesis, books, scientific articles and in different databases and the use of the family file. Conclusion; the main risk factors identified in this group of families were some diseases; allergies, diabetes, hypertension, due to polluting emissions and environmental conditions, inadequate nutrition, health risks, presence of industries, these risks could be reduced with proper care.
\end{abstract}

Keywords: Primary care, community nursing, family file, health risk factors. 


\section{Introducción.}

Un Análisis Situacional de Salud es un proceso de diagnóstico establecido por las autoridades del sistema de salud para recopilar información pertinente en una población determinada para así conocer información referente a la situación de las enfermedades que determinan el estado saludable de estos, forma parte del gran sistema organizado de atención de salud y es un componente importante para iniciar los planes en esta área.

De acuerdo al Ministerio de Salud del Ecuador el Análisis Situacional de Salud ASIS es una de las principales herramientas conceptuales y metodológicas para la implementación del Modelo (MAIS-FCI) en el que con la participación de los actores sociales se construye desde lo local el diagnóstico, se analiza la realidad, la identificación de problemas de salud, las prioridades y los medios para transformar esos problemas a través de la construcción de Planes Locales de Salud (PLS).

En este sentido con la implementación del ASIS se logra tener un acercamiento a las comunidades para así conocer el estado general de salud de sus habitantes, lo que significa un paso importante para la planificación en direccionar los recursos estatales y así solventar las necesidades de salud detectadas.

El análisis de situaciones de salud (ASIS) surge como respuesta a una visión de salud pública que reclama: complejidad, integralidad, eficacia y plena participación de todos los actores sociales en la gestión de los problemas que afectan la salud de la población. Es decir, el ASIS es resultado de la propia práctica de actuación en salud y; además, de la decisión de concebir la realidad cotidiana de un conjunto diverso y articulado de dimensiones y factores que influencian la prevalencia de determinadas dolencias (Orlando, Tulio, Guadalupe, \& Perdomo, 2016).

Este proyecto se ejecutará en lineamiento a los protocolos establecidos ante la situación actual por la pandemia del Covid-19, razón por la cual se limitará al diagnóstico de las familias de los autores, es decir de los profesionales en formación de la carrera de enfermería de tercer semestre de la Universidad Estatal del Sur de Manabí, los cuales se encuentran ubicados en diferentes puntos del país.

Por lo tanto, se beneficiará a los estudiantes con el conocimiento de la aplicación de este análisis que forma parte de sus competencias profesionales, a la vez que podrán detectar alguna anomalía en el estado de salud de sus familias, que son los beneficiarios secundarios, además de aportar a la literatura de la temática tratada.

En los grupos poblacionales pueden coexistir un gran número de dificultades que afectan el estado general, así como los servicios con los que cuenta y las amenazas presentes en el entorno, son variables que condicionan el estado de salud de una determinada población. Muchas veces el desconocimiento de estas variables incide en que no se enfoquen los recursos necesarios para atender todas las necesidades que presenta una comunidad.

A pesar de los esfuerzos del Ministerio de Salud Pública por brindar una atención más integral y con acceso para todos, aún quedan muchos lugares donde es necesario aplicar herramientas de diagnóstico situacional para conocer la realidad con la que viven los ciudadanos en el campo de salud conociendo sus determinantes que afectan su calidad de vida.

El incremento y la aparición de nuevas enfermedades o sucesos que puedan afectar la salud de los habitantes, por lo que existe la necesidad de mantener actualizados los datos de las poblaciones en cuanto a los índices de salud. Actualmente en el marco mundial de pandemia causada por el COVID-19, la población se encuentra en un periodo de cuarentena, incrementando las necesidades de salud, debilitando el sistema sanitario y que no permite cubrir la demanda que exige la población enferma.

La investigación se basa en la evaluación de un conjunto de familias situadas en las distintas provincias del país, cuyo factor en común es que uno de sus integrantes es parte de este trabajo; estudiantes del tercer semestre de la carrera de enfermería de la Universidad Estatal del Sur de Manabí, y conocer el estado situacional de salud de este grupo poblacional.

El Análisis Situacional Integral de Salud (ASIS) es una estrategia aplicada por los equipos de atención integral de salud, identificando numerosos factores basándose en lineamientos de análisis que ayudan a la conformación de este diagnóstico situacional. La implementación del ASIS es el primer paso para brindar una atención integral, sus resultados definen los planes de acción con lo que se trabajará en la comunidad y así hacer frente a las necesidades de la población de forma ordenada y coordinada conjuntamente con los representantes de ambas partes, conformándose el comité local de salud quienes se encargarán de socializar las estrategias a seguir para aplicar los planes locales de salud.

El ASIS busca contribuir con el mejoramiento de los procesos de conducción en salud mediante el establecimiento de perfiles de salud y sus determinantes, con énfasis en la identificación de las brechas de inequidad existentes; la 
identificación de prioridades de política, programas y planes de salud, identificación de territorios vulnerables, definición de las intervenciones costo-efectivas y factibles de implementación, evaluación y monitoreo de los planes y programas de salud, y la construcción de escenarios prospectivos en salud (Vicuña, 2016).

Teniendo en cuenta los diferentes enfoques y dentro del enfoque de determinante sociales, se considera la salud como un complejo social de múltiples circunstancias, siendo así el resultado de múltiples interacciones sociales, culturales, biológicas y políticas, factores presentes en el territorio, y que su desarticulación y/o articulación han jugado y juegan un papel importante en el deterioro de salud de las poblaciones (Mosquera, 2019).

El ASIS, estudia la interacción entre las condiciones de vida y el nivel de los procesos de salud en las diferentes escalas territoriales, políticas y sociales. Se incluye como fundamento el estudio de los grupos poblacionales con diferentes grados de desventaja, consecuencia de la distribución desigual de las condiciones de vida de acuerdo con el sexo, edad y nivel socioeconómico que se desarrollan en ambientes influenciados por el contexto histórico, geográfico, demográfico, social, económico, cultural, político y epidemiológico, en donde se producen relaciones de determinación y condicionamiento (Moreno, 2017).

Los ASIS, como parte de la Salud Pública, conllevan una intención de intervenir y dirigidos a fortalecer las decisiones, e involucran un compromiso con la transformación de la salud de las poblaciones y sobre todo, con la reducción de las inequidades sociales en salud (Grela, 2015). Que aun en la actualidad se mantienen.

Es una actividad necesaria en la atención pública de salud que tiene como propósito identificar las características socio psicológicas, económicas, históricas, geográficas, culturales y ambientales que inciden en la salud de la población, así como los problemas de salud que presentan los individuos, las familias, los grupos y la comunidad en su conjunto, para desarrollar acciones que contribuyan a su solución. La elaboración del ASIS constituye el elemento base para la planificación estratégica a ese nivel, y establece las prioridades, pues dispone de los recursos locales en función de ellos (Pría, Louro, Fariñas, Gómez, \& Segredo, 2016).

A su vez los ASIS se construyen articulando datos de fuentes primarias y secundarias dispersas en diferentes instituciones (no necesariamente del sector salud) o directamente de terreno que posean el común de permitir caracterizar la situación de salud de la población, en todos sus aspectos (GACBA, 2017).
Este instrumento está permitiendo medir la situación de salud de la población en sus distintas dimensiones (variables demográficas, sociales, económicas y epidemiológicas), a través de medidas representativas (promedios y otras medidas de tendencia central) y del establecimiento de brechas de inequidad para identificar los grupos más vulnerables. Esta información permitirá al sector acondicionar la oferta de servicios para enfrentar estratificada mente estos problemas, de forma que se obtenga mayor beneficio social, en términos de un mejor estado de salud (MINSAL, 2015).

El ASIS tiene también un carácter ético, dado por su condición de ser una investigación clínica, epidemiológica y social que se realiza en el primer nivel de atención. El ASIS y calidad de vida, es una práctica transformadora, y para que sea integral requiere tomar en cuenta a todos los subsistemas sociales posibles: antecedentes históricos de la población y los macro determinantes políticos, sociales, económicos, culturales, étnicos (Francis, 2016).

La finalidad del proyecto es conocer las determinantes de salud de las once familias de diversas provincias del país cuyo representante son los estudiantes de tercer semestre de la Carrera de Enfermería de la Universidad Estatal del Sur de Manabí, y mejorar el estado de salud de las familias ejecutando actividades que favorezcan su calidad de vida.

El proyecto brinda un doble beneficio para los estudiantes de enfermería, incrementando sus habilidades y conocimientos teóricos y prácticos a la vez que se trabaja en sus propias familias, identificando riesgos de salud que pudieran manifestarse y tomar acciones que les permita mitigar el impacto de las enfermedades.

El objetivo principal de la investigación, elaborar el diagnóstico situacional de estas familias, lo cual se logrará con tres componentes específicos: identificar los principales riesgos presentes en esto, mediante la aplicación de fichas familiares, describir el perfil epidemiológico de estas familias con la interpretación de los resultados y diseñar un plan enfocado en la prevención y mitigación de los factores de riesgo y enfermedades detectadas.

\section{Metodología}

La metodología utilizada la investigación es descriptiva, La información bibliográfica se obtuvo mediante la recopilación de información de fuentes confiables como tesis, libros, artículos científicos de las diferentes bases de datos. La técnica aplicada fue la ficha familiar, el formulario de APGAR familiar,

La unidad de análisis está compuesta por 11 familias de los estudiantes que conforman en proyecto, en total 53 personas. Criterios de inclusión: todos los familiares de los 11 
estudiantes que integraban el grupo de investigadores y que dieron su consentimiento en la participación. Criterios de exclusión: aquellos familiares que no brindaron in formación.

\section{Resultados}

Se presentan los resultados de las fichas familiares:

\section{Ubicación de las familias}

Tabla 1. Ubicación de las familias

\begin{tabular}{|c|c|c|}
\hline Alternativas & Frecuencia & Porcentajes \\
\hline Manabí & 6 & $54.55 \%$ \\
\hline Pichincha & 1 & $9.09 \%$ \\
\hline Santo Domingo & 1 & $9.09 \%$ \\
\hline Guayas & 1 & $9.09 \%$ \\
\hline Bolívar & 1 & $9.09 \%$ \\
\hline El Oro & 1 & $9.09 \%$ \\
\hline Total & 11 & $100 \%$ \\
\hline
\end{tabular}

Fuente: Fichas familiares de estudiantes

Elaborado por: Tercer semestre "A" Enfermería

\section{Análisis e interpretación}

Del total de las 11 familias el $54.55 \%$ es de la provincia de Manabí, es decir 7 familias, el resto Población están repartidos entre las provincias de Pichincha, Santo Domingo, Guayas, Bolívar, El Oro, una familia en cada provincia.

Tabla 2. Población

\begin{tabular}{|c|c|c|}
\hline Alternativas & Frecuencia & Porcentajes \\
\hline Manabí & 32 & $60.38 \%$ \\
Pichincha & 2 & $3.77 \%$ \\
Santo Domingo & 3 & $5.66 \%$ \\
Guayas & 3 & $5.66 \%$ \\
Bolívar & 9 & $16.98 \%$ \\
El Oro & 4 & $7.55 \%$ \\
\hline Total & 53 & $100 \%$ \\
\hline
\end{tabular}

Fuente: Fichas familiares de estudiantes

Elaborado por: Tercer semestre "A" Enfermería

\section{Análisis e interpretación}

En el total de la población, el $60.38 \%$ es de la provincia de Manabí, y le sigue Bolívar con el Edad

Tabla 3. Edad

\begin{tabular}{|c|c|c|}
\hline Alternativas & Frecuencia & Porcentajes \\
\hline Menos de 1 año & 0 & $0.00 \%$ \\
De 1 a 4 años & 4 & $7.55 \%$ \\
De 5 a 9 años & 7 & $13.21 \%$ \\
De 10 a 19 años & 12 & $22.64 \%$ \\
De 20 a 64 años & 30 & $56.60 \%$ \\
De 65 a más años & 0 & $0.00 \%$ \\
\hline Total & 53 & $100 \%$ \\
\hline
\end{tabular}

Fuente: Fichas familiares de estudiantes

Elaborado por: Tercer semestre "A" Enfermería

\section{Análisis e interpretación}

En la barra estadística de edad podemos encontrar que no hay personas menores de 1 año, de 1 a 4 años 4 personas que cumple el $7,55 \%$, de 5 a 9 años 7 personas que cumplen el $13,21 \%$, de 10 a 19 años 12
$16.98 \%$, el Oro con el $7.55 \%$, Guayas con el 5.66\%, Santo Domingo con el $5.66 \%$ y Pichincha con el $3.77 \%$ de todas las personas.

\section{Sexo}

Tabla 3. Sexo

\begin{tabular}{l|l|l} 
Alternativas & Frecuencia & Porcentajes \\
\hline
\end{tabular}




\begin{tabular}{|c|c|c|}
\hline Masculino & 26 & $49.06 \%$ \\
Femenino & 27 & $50.94 \%$ \\
\hline Total & 53 & $100 \%$ \\
\hline
\end{tabular}

Fuente: Fichas familiares de estudiantes

Elaborado por: Tercer semestre "A" Enfermería

\section{Análisis e interpretación}

En el total de la ficha familiar se pudo constatar que en las 10 familias existe un mayor porcentaje de Identificación étnica

Tabla 5. Identificación étnica

\begin{tabular}{|c|c|c|}
\hline Alternativas & Frecuencia & Porcentajes \\
\hline Mestizos & 23 & $43.40 \%$ \\
Montubios & 21 & $39.62 \%$ \\
Indígenas & 9 & $16.98 \%$ \\
\hline Total & 53 & $100 \%$ \\
\hline
\end{tabular}

Fuente: Fichas familiares de estudiantes

Elaborado por: Tercer semestre "A" Enfermería

\section{Análisis e interpretación}

Es evidente que la mayor parte de las personas que se presentan en la ficha familiar son mestizos que lo conforman 23 personas y cumple el $43,40 \%$ del total, la parte media son montubios que lo conforman 21 mujeres que cumplen el $50,94 \%$ y un porcentaje de hombre que cumple el $49,06 \%$ con una diferencia mínima para su balance de hombres y mujeres. personas y cumplen el $39,62 \%$ del total, la menor parte son indígenas que lo conforman 9 personas y cumple el $16,98 \%$ del total, sumando todo esto daría el numero de 53 personas que es el total de las personas que conforman las 10 familias de las diferentes partes del Ecuador.

\section{Ocupación}

Tabla 6. Ocupación

\begin{tabular}{|c|c|c|}
\hline Alternativas & Frecuencia & Porcentajes \\
\hline Estudiante & 28 & $52.83 \%$ \\
Ama de casa & 5 & $9.43 \%$ \\
Comerciante & 3 & $7.55 \%$ \\
Soldador & 1 & $1.89 \%$ \\
Jornalero & 1 & $1.89 \%$ \\
Agricultor & 1 & $1.89 \%$ \\
Auxiliar de limpieza & 1 & $1.89 \%$ \\
Mecánico & 2 & $3.77 \%$ \\
Albañil & 1 & $1.89 \%$ \\
Chofer & 1 & $1.89 \%$ \\
Profesionales & 2 & $3.77 \%$ \\
Ninguna & 2 & $11.32 \%$ \\
\hline Total & 53 & $100 \%$ \\
\hline
\end{tabular}

Fuente: Fichas familiares de estudiantes

Elaborado por: Tercer semestre "A” Enfermería

\section{Análisis e interpretación}

Se puede mostrar en las barras que la mayor parte de los integrantes de las 10 familias son estudiantes llegando a un total de 28 estudiantes que cumple el $52,83 \%$, amas de casa 5 personas que cumplen con el $9,43 \%$, comerciantes 3 personas que cumple el $7,55 \%$, soldado 1 persona que cumple con el $1,89 \%$, jornalero 1 persona que cumple con el 1,89\%, agricultor una persona que cumple con $1,89 \%$, auxiliar de limpieza 1 persona que cumple con el $1,89 \%$, mecánico 1 persona que cumple con el 1 , 895 , albañil 1 persona que cumple con el $1,89 \%$, chofer 1 persona que cumple con el $1,89 \%$, profesionales 2 personas que cumplen con el 3,77\%, desempleados 2 personas que cumplen el $11,32 \%$, y podríamos decir que la mayor parte de la población son estudiantes.

\section{Escolaridad}

Tabla 7. Escolaridad

\begin{tabular}{l|l|l|} 
Alternativas & Frecuencia & Porcentajes
\end{tabular}




\begin{tabular}{|c|c|c|}
\hline Sin escolaridad & 4 & $7.55 \%$ \\
Educación básica & 19 & $35.85 \%$ \\
Bachillerato & 17 & $32.08 \%$ \\
Superior & 11 & $20.75 \%$ \\
Especialización & 2 & $3.77 \%$ \\
\hline Total & 53 & $100 \%$ \\
\hline
\end{tabular}

Fuente: Fichas familiares de estudiantes

Elaborado por: Tercer semestre "A" Enfermería

\section{Análisis e interpretación}

Entre los niveles de estudio podemos encontrar que hay 4 personas que son analfabéticas que cumplen el $7,55 \%, 19$ personas que solo llegaron hasta educación básica que cumple el 35,85\%, 17 personas que son bachilleres que cumplen el $32,08 \%, 11$ personas con educación superior que cumplen el Vacunas

Tabla 8. Vacunas

\begin{tabular}{|c|c|c|}
\hline Alternativas & Frecuencia & Porcentajes \\
\hline Si & 50 & $94.34 \%$ \\
No & 3 & $5.66 \%$ \\
\hline Total & 53 & $100 \%$ \\
\hline
\end{tabular}

Fuente: Fichas familiares de estudiantes

Elaborado por: Tercer semestre "A" Enfermería

\section{Análisis e interpretación}

Podemos verificar que hay un total de 50 personas que, si cumplió con el esquema de vacunación que cumple el $94,34 \%$, un total de 3 personas que no cumplieron con el esquema de vacunación que cumple el $5,66 \%$, estas tres personas que no Salud Bucal

Tabla 9. Salud Bucal

\begin{tabular}{|c|c|c|}
\hline Alternativas & Frecuencia & Porcentajes \\
\hline Si & 47 & $88.68 \%$ \\
No & 6 & $11.32 \%$ \\
\hline Total & 53 & $100 \%$ \\
\hline
\end{tabular}

Fuente: Fichas familiares de estudiantes

Elaborado por: Tercer semestre "A" Enfermería

\section{Análisis e interpretación}

En este cuadro de barras se puede ver que hay un total de 47 personas que cumple con una buena salud bucal que es el $88,68 \%$, un total de 6 personas que Mortalidad

Tabla 10. Mortalidad

\begin{tabular}{|c|c|c|}
\hline Alternativas & Frecuencia & Porcentajes \\
\hline Si & 1 & $1.89 \%$ \\
No & 10 & $20.75 \%$ \\
\hline Total & 11 & $100 \%$ \\
\hline
\end{tabular}

Fuente: Fichas familiares de estudiantes

Elaborado por: Tercer semestre "A" Enfermería

\section{Análisis e interpretación}

Una de las familias representó mortalidad de dos de sus integrantes, uno falleció a causa del COVID-19 no cumple con una buena salud bucal que cumple con el $11,32 \%$, decimos que una buena salud bucal es muy importante ya nos brinda una mejor armonía y tenemos una buena presentación bucal.
20,75\%, 2 personas especializadas que cumplen el $3,77 \%$, podemos ver que la mayor parte de las personas de esta familias están en educación básica ya que personas adultas han llegado hasta educación básica y menores de edad que están en procesos de superación en la educación. cumplieron con el esquema de vacunación está en un riesgo de padecer enfermedades con más frecuencia ya que es muy importante aquello porque a través de ello estamos más protegidos de algunas enfermedades.

\section{Morbilidades}

Tabla 11. Morbilidades

\begin{tabular}{|c|c|c|}
\hline Alternativas & Frecuencia & Porcentajes \\
\hline Ninguna & 30 & $56.60 \%$ \\
Alergias & 7 & $13.21 \%$ \\
\hline
\end{tabular}

y otro por bronquitis, el resto de familias no presenta mortalidad, por lo tanto, el porcentaje de mortalidad en el grupo de familias se ubica en $1.89 \%$. 


\begin{tabular}{|c|c|c|}
\hline Dermatitis atópica & 2 & $3.77 \%$ \\
Discapacitado & 2 & $3.77 \%$ \\
Gastritis & 1 & $1.89 \%$ \\
Hepatitis & 1 & $1.89 \%$ \\
Adenoides & 1 & $1.89 \%$ \\
Sobrepeso & 1 & $1.89 \%$ \\
Rinitis & 1 & $1.89 \%$ \\
Reumatismo & 1 & $1.89 \%$ \\
Diabetes & 1 & $1.89 \%$ \\
Eritemas & 1 & $1.89 \%$ \\
Espolón Calcáneo & 1 & $1.89 \%$ \\
Hipertensión & 1 & $1.89 \%$ \\
Asma & 1 & $1.89 \%$ \\
Taquicardia & 1 & $1.89 \%$ \\
\hline Total & 53 & $100 \%$ \\
\hline
\end{tabular}

Fuente: Fichas familiares de estudiantes

Elaborado por: Tercer semestre "A" Enfermería

\section{Análisis e interpretación}

Existe un total de 30 personas que no padecen de una enfermedad o patología, 7 personas alérgicas que cumplen el 13,21\%, 2 personas con dermatitis atópica que cumplen el 3,77\%, 2 personas discapacitadas que cumplen con el 3,77\%, 1 persona con gastritis que cumple con el 1,89\%, 1 persona con hepatitis que cumple el $1,89 \%, 1$ persona con adenoides que cumple el $1,89 \% 1$ persona con sobrepeso que cumple con el $1,89 \%, 1$ persona con rinitis que cumple con el 1,89\%, 1 persona con reumatismo que cumple con el $1,89 \%, 1$ persona con diabetes que cumple con el 1,89\%, 1 persona con eritema que cumple con el 1,89\%, 1 persona con espolón calcáneo que cumple con el 1,89\%, 1 persona con hipertensión que cumple con el 1,89\%, 1 persona con asma que cumple con el 1,89\%, 1 persona con taquicardia que cumple con el $1,89 \%$, existen enfermedades y patologías que afectan a la mayoría de las personas de estas 10 familias y tienen un gran riesgo de complicaciones.

\section{Apgar familiar}

Tabla 12. Apgar familiar

\begin{tabular}{|c|c|c|}
\hline Alternativas & Frecuencia & Porcentajes \\
\hline Alta satisfacción: buena función familiar & 6 & $54.54 \%$ \\
Mediana satisfacción: disfunción leve & 3 & $27.27 \%$ \\
Baja satisfacción: disfunción moderada & 2 & $18.18 \%$ \\
Baja satisfacción: disfunción severa & 0 & $0.00 \%$ \\
\hline Total & 11 & $100 \%$ \\
\hline
\end{tabular}

Fuente: Fichas familiares de estudiantes

Elaborado por: Tercer semestre "A" Enfermería

\begin{abstract}
Análisis e interpretación
En esta tabla de barras encontramos los resultados del APGAR familiar donde decimos que hay un total de 6 personas con una alta satisfacción familiar (buena función familiar) que cumple con el 54,54\%, un total de 6 personas con una mediana satisfacción
\end{abstract}

familiar ( difusión leve en la familia) que cumple el $27,27 \%$, un total de 2 personas con una baja satisfacción ( difusión moderada familiar) que cumple con el $18,18 \%$, existe un mayor de personas con una buena satisfacción familiar donde existe una buena relación, buena comunicación y una buena interrelación entre ellos.

\section{Riesgos biológicos}

Tabla13. Riesgos biológicos

\begin{tabular}{|l|c|c|}
\hline \multicolumn{1}{|c|}{ Alternativas } & Frecuencia & Porcentajes \\
\hline Familias con personas que presenten vacunación incompleta & 2 & $18.18 \%$ \\
Familias con personas que presenten malnutrición & 3 & $27.27 \%$ \\
Familias con personas que presenten enfermedad de impacto & 5 & $45.45 \%$ \\
Familias con personas que presenten discapacidad & 1 & $9.09 \%$ \\
\hline \multicolumn{1}{|c|}{ Total } & 11 & $100 \%$ \\
\hline
\end{tabular}


Fuente: Fichas familiares de estudiantes

Elaborado por: Tercer semestre "A" Enfermería

\section{Análisis e interpretación}

En los riesgos biológicos con el que más presencia se pudo encontrar son las familias con personas que presenten enfermedades de impacto con el $45.45 \%$ de estos riesgos, le siguen las familias con personas que presenten malnutrición con el $27.27 \%$, y le siguen las familias con personas que presenten vacunación incompleta con el $18.18 \%$ y las familias con personas que presenten discapacidad que representa el $9.09 \%$ de estos riesgos.

\section{Riesgos sanitarios}

Tabla 4. Riesgos sanitarios

\begin{tabular}{|l|c|c|}
\hline \multicolumn{1}{|c|}{ Alternativas } & Frecuencia & Porcentajes \\
\hline Impacto ecológico por industrias & 1 & $9.09 \%$ \\
Consumo de agua insegura & 2 & $18.18 \%$ \\
Animales intradomiciliarios & 8 & $72.73 \%$ \\
\hline \multicolumn{1}{|c|}{ Total } & 11 & $100 \%$ \\
\hline
\end{tabular}

Fuente: Fichas familiares de estudiantes

Elaborado por: Tercer semestre "A" Enfermería

\section{Análisis e interpretación}

En los riesgos sanitarios solamente se encontraron tres de estos, el impacto ecológico por industrias que corresponde al $9.09 \%$ de estos, el consumo de agua insegura que corresponde al $18.18 \%$ y la presencia de animales intradomiciliarios con el $72.73 \%$.

\section{Riesgos socioeconómicos}

Tabla 5. Riesgos socioeconómicos

\begin{tabular}{|c|c|c|}
\hline Alternativas & Frecuencia & Porcentajes \\
\hline Pobreza & 3 & $27.27 \%$ \\
\hline Analfabetismo del padre o la madre & 5 & $45.45 \%$ \\
\hline Malas condiciones de la vivienda & 2 & $18.18 \%$ \\
\hline Hacinamiento & 1 & $9.09 \%$ \\
\hline Total & 11 & $100 \%$ \\
\hline
\end{tabular}

Fuente: Fichas familiares de estudiantes

Elaborado por: Tercer semestre "A" Enfermería

\section{Análisis e interpretación}

En los riesgos socioeconómicos se identificaron 4 riesgos, de los cuales el mayor son analfabetismo del padre o la madre que corresponde al $45.45 \%$ de estos, también se encontró pobreza en un $27.27 \%$, malas condiciones de la vivienda en un $18.18 \%$, y hacinamiento con otro $9.09 \%$.

\section{Valoración general de los riesgos}

Tabla 6. Valoración general de los riesgos

\begin{tabular}{|c|c|c|}
\hline Alternativas & Frecuencia & Porcentajes \\
\hline Sin riesgo & 0 & $0 \%$ \\
Bajo riesgo & 11 & $100 \%$ \\
Medio riesgo & 0 & $0 \%$ \\
Alto riesgo & 0 & $0 \%$ \\
\hline Total & 11 & $100 \%$ \\
\hline
\end{tabular}

Fuente: Fichas familiares de estudiantes

Elaborado por: Tercer semestre "A" Enfermería

\section{Análisis e interpretación}

En la valoración general de los riesgos el 100\% de las familias presenta un bajo riesgo.

\section{Conclusiones}

Los principales factores de riesgo identificados en este grupo de familias tienen un nivel de riesgo bajo y se manifiestan especialmente en el surgimiento de algunas enfermedades comunes, como alergias, diabetes, hipertensión, debido a emisiones contaminantes y condiciones del entorno, mala

alimentación estos riesgos se podrían disminuir con los cuidados adecuados.

Las enfermedades que prevalecen en el conjunto de familias evaluadas dentro del perfil epidemiológico son, alergias, diabetes, hipertensión, dermatitis atópica derivadas quizás de la presencia de agentes 


\section{HIGIA DE LA SALUD}

contaminantes en el entorno representado en los riesgos sanitarios detectados, y de industrias cercanas a las viviendas que estarían incidiendo en este tipo de enfermedades.

A través del plan local enfocado en la prevención y mitigación de los riesgos detectados a partir de las fichas familiares, se ejecutaron por la organización familiar, y el aporte de los profesionales en
ISSN 2773-7705

Periodo. Julio - Diciembre 2019

Vol. 1, Nro. 1, Publicado 2019-12-31

formación y con la oportuna gestión del comité familiar de salud, se educó a las personas con charlas, mingas, talleres, videos, que mejorar el estado de salud de este grupo de familias ecuatorianas.

\section{Colaboradores de la Investigación:}

Lissette María Saltos Peñaherrera; Tatiana Monserrathe Espinoza Zambrano; Andrés Alejandro Coello Montaquiza; Kevin Stiveen Caguana Orriola; Ángel Javier Santana González; Mirian Verónica Pasto Patín; Michelle Cecibel Nevarez Rodríguez; Andy Javier Cedeño Castro; Dayana Kasandra Bailón Figueroa; Shirley Vanessa Sánchez Mantuano; Víctor Adrián Alonso Mantuano.

Universidad estatal del Sur de Manabí, Carrera de enfermería, estudiantes de cuarto semestre.

\section{Referencia bibliográfica}

Francis, M. (2016). Analisis de salud ASIS. Hubspot.

GACBA. (2017). Análisis de Situación de Salud (ASIS) . Gobierno de la Ciudad de Buenos Aires.

GoRaymi. (2016). Historia del Ecuador. Recuperado el Agosto de 2020, de Historia del Ecuador: https://www.goraymi.com/es-ec/ecuador/historias/historia-ecuador-a2rmy00r8

Grela, M. (2015). Análisis Situacional de Salud. Universidad Nacional de Córdoba, Argentina.

MINSAL. (2015). Guía para el análisis del proceso salud-enfermedad. ASIS . Ministerio de Salud de Perú.

Moreno, C. (2017). Análisis de situación de salud ASIS. Gobierno de Colombia, Dirección de Epidemiología y Demografía, Colombia.

Mosquera, M. (2019). Actualización Análisis de Situación de Salud (ASIS) 2018con el ModeloDe los Determinantes Sociales de Salud. Secretaría de Salud Departamental.

Orlando, J., Tulio, M., Guadalupe, M., \& Perdomo, R. (2016). Análisis de Situación de Salud según condiciones de vida. Secretaría de Salud de Honduras.

Pría, M., Louro, I., Fariñas, A., Gómez, H., \& Segredo, A. (2016). Guía para la elaboración del análisis de la situación de salud en la atención primaria. Revista Cubana de Medicina General Integral, 22(3).

RELASEDOR. (2016). Ecuador. Información País. FLACSO, Quito, Ecuador.

Vicuña, M. (2016). Guía Metodológica para el Análisis de Situación de Salud. Digepisalud. 